\title{
Validation of a Polyvinylidene Fluoride Impedance Sensor for Respiratory Event Classification during Polysomnography
}

\author{
Brian B. Koo, M.D. '; Colin Drummond, Ph.D. 2; Susan Surovec, B.A."; Nathan Johnson, M.S. .; Stephanie A. Marvin; \\ Susan Redline, M.D., M.P.H. ${ }^{3,4}$ \\ ${ }^{1}$ Case Western Reserve University School of Medicine; Department of Neurology, Cleveland, OH; \\ ${ }^{2}$ Case Western Reserve University, Department of Biomedical Engineering, Cleveland, $\mathrm{OH} ;{ }^{3}$ Brigham and Women's \\ Hospital and Beth Israel Deaconess Medical Center, Harvard Medical School, Department of Medicine, Boston, MA; \\ ${ }^{4}$ Case Western Reserve School of Medicine, Center for Clinical Investigation, Cleveland, $\mathrm{OH}$
}

Study Objectives: The AASM has recommended specific sensors in measuring apnea and hypopnea based on published reliability and validity data. As new technology emerges, these guidelines will need revision. Polyvinylidene fluoride (PVDF) measures impedance and can be incorporated into a belt to approximate airflow and respiratory effort. We compared respiratory event detection using PVDF impedance belts (PVDFb), respiratory inductance plethysmography (RIP), and nasal-oral pneumotachography (PNT).

Methods: First, in a clinical setting, 50 subjects (median AHI 26) undergoing polysomnography were fitted with PVDFb and standard sensors. Studies were scored in 4 independent passes using 4 respiratory montages (M); $\mathrm{M1}$ : nasal pressure transduction (NPT), thermistry, and RIP; M2: NPT, thermistry, and PVDFb; M3: thermistry and PVDFb; M4: PVDFb alone. Each experimental montage (M2-M4) was compared to the reference standard (M1) for total apneas and hypopneas. In a second experimental study, respiratory event detection was compared across a series of breathing trials for PVDFb, RIP, and PNT in normal subjects. Agreement was evaluated with intraclass correlation coefficient (ICC), $\mathrm{k}$ statistics, and Bland-Altman plots.

Results: ICCs comparing event numbers by $\mathrm{M} 1$ to $\mathrm{M} 2,3$, and 4 were: $0.99,0.93$, and 0.91 , respectively. Almost identical numbers of events were identified for M 1 and M2 (177.5 \pm 122.7 vs $177.6 \pm 123.2$ ). Event subtypes also were comparable. PVDFb was less sensitive than PNT but no different than $\mathrm{RIP}$ in detecting decreased breathing amplitude.

Conclusions: PVDFb was comparable to standard RIP in determining respiratory events during polysomnography and in detecting decreased breathing amplitude, suggesting that PVDFb can be used as an alternative to RIP for apnea/hypopnea evaluation.

Keywords: Sleep apnea, respiratory effort, signal, technology, sleep, validity

Citation: Koo BB; Drummond C; Surovec S; Johnson N; Marvin SA; Redline $S$. Validation of a polyvinylidene fluoride impedance sensor for respiratory event classification during polysomnography. J Clin Sleep Med 2011;7(5):479-485.
$\mathrm{T}$ he sleep related breathing disorders are characterized by repetitive episodes of complete or partial airflow cessation which result in apnea or hypopnea, respectively. Chief among these breathing disorders is obstructive sleep apnea (OSA) which affects approximately $9 \%$ of women and $24 \%$ of men in the general population. ${ }^{1}$ The principal metric reflecting OSA severity, the apnea-hypopnea index (AHI), quantifies the number of apneas and hypopneas per hour of sleep. The AHI has proven a valuable measure, as it correlates with daytime sleepiness, risk of cardiovascular disease, and mortality. ${ }^{2-5}$ For this reason, there has been much emphasis placed on methods for ensuring the reproducibility and validity of apnea and hypopnea detection.

In 2007, the American Academy of Sleep Medicine (AASM) published recommendations for measuring apneas and hypopneas based on evidence on the reliability and validity of alternative sensors and scoring approaches and consensus opinion. ${ }^{6}$ In this effort, technology reviewed for the estimation of airflow included the heat-sensitive thermistor, nasal pressure transduction (NPT), and respiratory inductance plethysmography (RIP). Based upon available evidence, thermistry was deemed ap-

\section{BRIEF SUMMARY \\ Current Knowledge/Study Rationale: The purpose of this study was to compare newer PVDF technology to gold standard technology in the identification of apnea and hypopnea. As new technology emerges, vali- dation studies become crucial so that new technology can not only be introduced but gain wider acceptance if appropriate. \\ Study Impact: Overall comparability for detecting respiratory distur- bances was demonstrated when using PVDF compared to currently recommended sensors.}

propriate for detecting apnea, while use of either NPT or RIP was recommended to identify hypopnea. It was recognized that distinguishing obstructive from central respiratory events requires identification of respiratory effort, which might be best measured by esophageal manometry. However, because of the burden and limited availability of this technique, RIP was recommended as an acceptable means for measuring breathing effort, ${ }^{7}$ noting that data regarding the use of RIP to measure respiratory effort are limited. ${ }^{8}$

The AASM recognized that recommendations might change as technologies evolved and new data were generated. 
A newer technology, for which there were not sufficient data available at the time of the initial AASM review, concerned use of polyvinylidene fluoride (PVDF) for measuring changes in airflow and effort. PVDF is a specialty fluoropolymer substance which reacts almost instantaneously to changes in temperature, pressure, strain, and impedance, making it a potentially useful substrate to sense respiratory flow or effort. ${ }^{9-11}$ Similar to inductance plethysmography, PVDF can be incorporated into a belt surrounding the chest and abdomen but unlike RIP, PVDF measures impedance and not inductance to estimate breathing and respiratory effort. Use of inductance technologies for respiratory measurement is based on the principle that the changes in current in the coiled wires surrounding the chest or abdomen induced by breathing are linearly proportional to changes in the cross-sectional areas occurring during breathing. In contrast, impedance measures changes in electrical resistance, which usually are not linearly related to changes in cross-sectional dimensions. ${ }^{12-14}$

Consistent with evidence-based guidelines on sensor evaluation, we evaluated the comparability of respiratory event and effort detection when using PVDF impedance belts (PVDFb) compared to currently recommended sensors, including RIP, NPT, heat-sensitive thermistry, and nasal-oral pneumotachography (PNT).

\section{METHODS}

Sensors were studied in a 2-step fashion. First, PVDFb sensors were added to a standard polysomnographic montage in patients presenting for clinical study to assess comparability of PVDFb to RIP in scoring and classification of apnea (central vs. obstructive) and comparability of PVDFb to NPT in scoring of hypopnea and apnea. Second, PVDFb sensors were used along with RIP and pneumotachography in normal volunteers to assess comparability of event detection when using PVDFb to a reference standard airflow measure, PNT. Studies were approved by the Institutional Review Board of University Hospitals Case Medical Center. Subjects provided written informed consent.

\section{Comparability of Respiratory Event Identification: Clinical Montages}

Fifty subjects (27 women) referred for evaluation of obstructive sleep apnea were included. Subjects were fitted with abdominal and thoracic PVDFb (Dymedix Corporation) in addition to standard sensors: NPT (Protech), thermistry (Embla), and RIP bands (Embla). Digital polysomnography (Embla) was performed using central and occipital electroencephalography (EEG), right and left electrooculography (EOG), chin and bilateral pretibial electromyography (EMG), electrocardiography (ECG), and arterial oxygen saturation. Sleep and arousal were scored manually, using standard criteria. ${ }^{7}, 15$

For each study, respiratory events were scored by a single scorer using 4 alternative viewing montages: (1) montage 1 (reference standard): NPT, thermistry, and RIP (chest, abdomen, sum); (2) montage 2: NPT, thermistry, and PVDFb (chest, abdomen, sum); (3) montage 3: thermistry and PVDFb (chest, abdomen, sum); (4) montage 4: PVDFb (chest, abdomen, sum). The scorer was blinded to the specific signal identity for RIP and PVDFb in montages 1 and 2, but NPT and thermistry were labeled when used and PVDFb channels were labeled in montages 3 and 4 . Additionally, each montage included information from the hypnogram regarding sleep stages, marked arousals, and arterial oxygen saturation. Scoring was carried out manually on an epoch by epoch basis, with each montage presented in random order while avoiding consecutive presentations of montages from the same study.

Apnea was defined as $<10 \%$ fluctuation of airflow signal from thermistry in montage 1,2 , and 3 , and absence of signal in PVDFb in montage 4 for $\geq 10 \mathrm{sec}$. Apnea was defined as obstructive or central when breathing effort persisted or was absent, respectively. Effort was determined by RIP in montage 1 and by PVDFb in montages 2 and 3. In montage 4, central apnea and hypopnea were scored; in this montage, no attempt was made to distinguish obstructive apnea from hypopnea. Hypopnea was defined as $>50 \%$ reduction in airflow from NPT in montages 1 and 2 and PVDFb sum in montages 3 and 4 . There was no desaturation or arousal requirement to score hypopnea. A random sample of 30 studies was rescored from each of the first 3 montages to assess intrascorer reliability.

Signal quality for each relevant signal for each montage was rated using an approach developed by our Sleep Reading Center. ${ }^{16}$ The amount of time with artifact-free signal was determined with following numerical conventions: (1) Poor: signal good $<25 \%$ of total sleep time (TST); (2) Fair: signal good $25 \%$ to $49 \%$ of TST; (3) Good: signal good $50 \%$ to $74 \%$ of TST; (4) Very Good: signal good $75 \%$ to $94 \%$ of TST; and (5) Excellent: signal good $>95 \%$ of TST.

\section{Quantitative Measurements: Reference Standard}

In the second study, 10 volunteers free of cardiopulmonary disease were recruited. Subjects were fitted with PVDFb (Dymedix Corporation), RIP (Embla), and with a face mask connected to a pneumotachograph (PNT) (Hans Rudolf 3830 Series, Kansas City, MO). Subjects were asked to breathe normally while awake and supine, following a breathing script which included: breaths augmented in volume, breaths with decreased volume, and breaths simulating both obstructive and central apnea. For each subject there was approximately $30 \mathrm{~min}$ of breathing data available for analysis. Prior to recording, PNT output was calibrated so that computed airflow volume corresponded with a precision 3-liter syringe (Hans Rudolf 5570 Series Syringe, Kansas City, MO).

The signals from the PNT pressure differentials were converted into $\mathrm{a} \pm 2.0 \mathrm{~V}$ analog signal through a highly sensitive pressure transducer (Honeywell DC010NDC4), recorded, and stored with a data acquisition system (National Instruments, VI software and SCB-68 interface, Austin, TX). Output signals from RIP and PVDFb were acquired using a standard Embla N700 system.

Data were exported to a European Data Format (EDF) file and then converted to a time-coded ACSII format. PNT signals were also exported from the NI system into a similar timecoded format so that the signals could be synchronized. For each subject, breathing data from PNT, RIP, and PVDFb were reviewed and scored independently of each other. Episodes of $50 \%$ reduction in signal amplitude $\geq 10 \mathrm{sec}$ were annotated for each different signal: PNT, RIP, and PVDFb. 


\section{Statistical Analysis}

\section{Comparability of Respiratory Event Identification}

\section{CLINICAL MONTAGES}

Results from the reference standard montage 1 were compared to each of the 3 other montages. The mean, median, standard deviation, and absolute differences (between each set of studies) for the number of obstructive apneas, central apneas, and hypopneas were determined and compared with a Wilcoxon test. Data from the reference standard montage 1 were compared to each of the 3 other montages. Bland-Altman plots were constructed for each pairwise comparison to assess bias. The intraclass correlation coefficients (ICC) and their 95\% confidence intervals and Spearman correlation coefficients were computed to continuous measures (absolute number of apneas and hypopneas). In addition, levels of agreement for dichotomous cutoffs of $\geq 5$ events per hour for disease classification for event indexes were assessed using the $\kappa$ statistic. Kappa $>0.70$ or ICC $>0.85$ was considered evidence of excellent agreement.

\section{Quantitative Measurements: Reference Standard}

The mean, standard deviation, and absolute differences (between each sensor) for the number of detected respiratory events were determined and compared with a Wilcoxon test. Bland-Altman plots were constructed for each pairwise comparison to assess bias. Kappa statistics $(\kappa)$ and intraclass correlation coefficients (ICC) and their 95\% confidence intervals were constructed to compare overall level of agreement in continuous measure (absolute number of respiratory events).

\section{RESULTS}

\section{Comparability of Respiratory Event Identification: Clinical Montages}

Subjects included 27 women and 23 men, the majority of whom were obese (mean BMI $36.2 \pm 8.2$ ) and had obstructive sleep apnea in the moderate range (median AHI 26.0 [15.8, 53.2; interquartile]). The average recording time per subject was $429 \pm 68$ minutes.

Figures 1A and $\mathbf{B}$ show examples of obstructive apnea as identified by thermistry plus nasal pressure transduction along with either RIP or PVDFb, respectively. In Figure 1C, hypopnea identified by PVDFb sum with the 50\% reduction amplitude criterion is depicted.

The distributions of respiratory events scored for each montage is shown in Table 1. There were virtually no differences in the total number of events scored or classification of events when PVDFb was used instead of RIP for event classification (i.e., M1 vs M2). Fewer events were detected when NPT was eliminated from the montage (M3 and M4). The further removal of the thermistor in M4, where by definition, events could not be classified as obstructive apneas, resulted in a further reduction in total respiratory events although no difference in hypopnea detection as compared to M3.

\section{Comparison of Montage 1 and 2: Influence of Using PVDFb vs RIP}

Table 2 provides a further comparison of scoring results for montages 1 and 2. Since these 2 montages included thermistry and NPT but differed in how effort was determined (i.e., RIP in montage 1 and PVDFb in montage 2), this comparison assesses the comparability of PVDFb to RIP in detecting respiratory effort for the purpose of distinguishing obstructive and central apneas. Compared to montage 1 (reference standard), there were only small differences in the total number

\section{Figure 1}

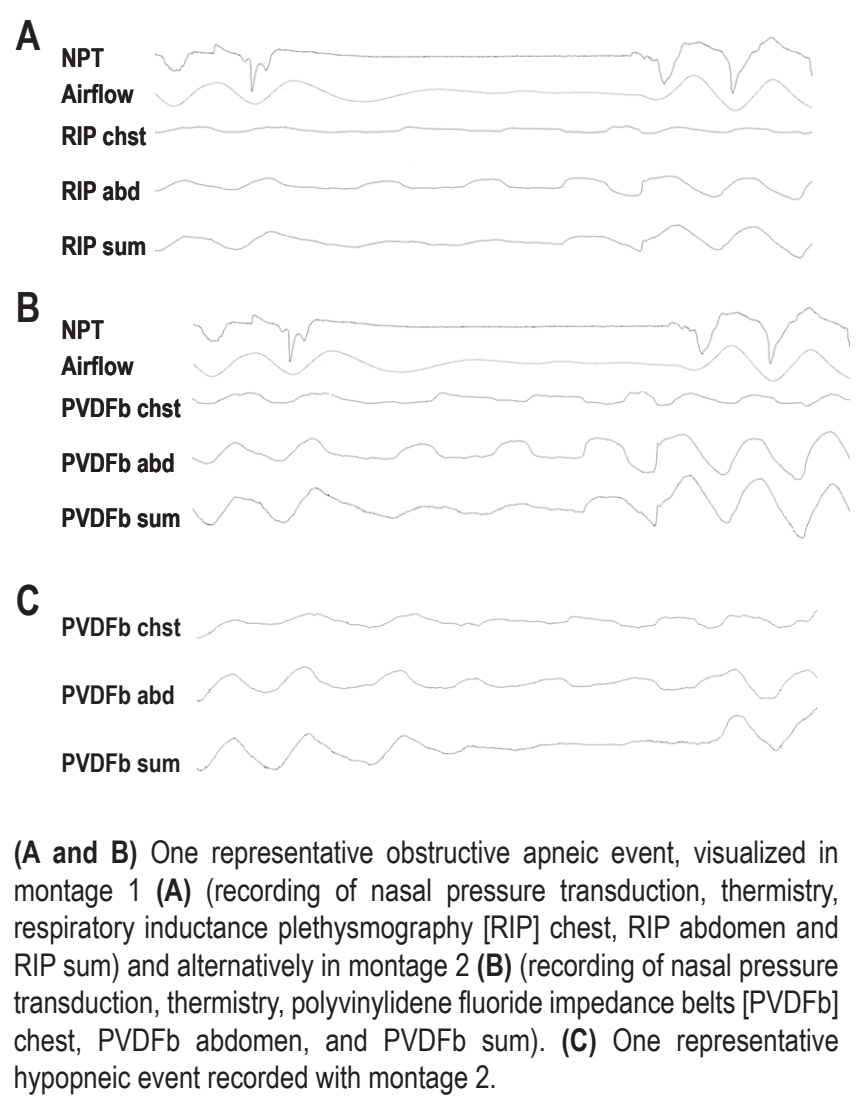

Table 1-Total number of respiratory events scored per study for each montage

\begin{tabular}{|c|c|c|c|c|c|c|}
\hline \multirow[b]{2}{*}{ Obstructive Apnea } & \multirow{2}{*}{$\frac{\text { Montage } 1(n=50)}{17.4 \pm 36.4}$} & Montage $2(n=50)$ & \multicolumn{2}{|c|}{ Montage $3(n=50)$} & \multicolumn{2}{|c|}{ Montage $4(n=50)$} \\
\hline & & $16.6 \pm 35.2 \quad P=0.87$ & $13.8 \pm 34.1$ & $P=0.50$ & - & - \\
\hline Central Apnea & $13.1 \pm 41.8$ & $13.0 \pm 41.2$ & $13.5 \pm 41.9$ & $P=0.93$ & $13.9 \pm 43.2$ & $P=0.96$ \\
\hline Hypopnea & $160.0 \pm 111.6$ & $161.0 \pm 114.3 P=0.96$ & $123.3 \pm 93.7$ & $P=0.07$ & $122.0 \pm 99.4$ & $P=0.07$ \\
\hline Obstructive Apneas \& Hypopneas & $177.5 \pm 122.7$ & $177.6 \pm 123.2 P=0.95$ & $137.1 \pm 102.8$ & $P=0.09$ & $122.0 \pm 99.4$ & $P=0.02$ \\
\hline
\end{tabular}

Mean \pm SD. P-value from Wilcoxon test comparing to Montage 1 . 
Table 2-Comparison of number of events scored per study in Montage 1 vs. Montage 2: impact of substituting PVDFb for RIP

$\begin{array}{lcccc} & \begin{array}{c}\text { Difference }(\mathrm{M} 2-\mathrm{M} 1) \\ (\text { mean } \pm \text { SD) }\end{array} & \begin{array}{c}\text { Spearman } \\ \text { correlation }\end{array} & \begin{array}{c}\text { ICC } \\ (95 \% \mathrm{CI})\end{array} & \begin{array}{c}\text { Kappa } \\ \text { statistic }\end{array} \\ \text { Obstructive Apnea } & -0.8 \pm 9.6 & 0.940 & 0.964(0.937,0.979) & 0.935 \\ \text { Central Apnea } & -0.1 \pm 1.5 & 0.953 & 0.999(0.999,1.00) & 0.898 \\ \text { Hypopnea } & 0.9 \pm 16.0 & 0.985 & 0.990(0.982,0.994) & 0.847 \\ \text { Obstructive Apneas \& Hypopneas } & 0.1 \pm 14.1 & 0.987 & 0.993(0.988,0.996) & 0.847\end{array}$

Mean \pm SD. Kappa statistics comparing montage 2 to montage 1 for sleep apnea severity based on the event index (number of events per hour of sleep) $\geq 5$.

Table 3-Comparison of number of events scored per study in Montage 1 vs. Montage 3

$\begin{array}{lcccc} & \begin{array}{c}\text { Difference }(\text { M3-M1) } \\ \text { (mean } \pm \text { SD) }\end{array} & \begin{array}{c}\text { Spearman } \\ \text { correlation }\end{array} & \begin{array}{c}\text { ICC } \\ (95 \% \text { Cl) }\end{array} & \begin{array}{c}\text { Kappa } \\ \text { statistic }\end{array} \\ \text { Obstructive Apnea } & -3.6 \pm 10.0 & 0.942 & 0.959(0.929,0.977) & 0.929 \\ \text { Central Apnea } & 0.4 \pm 2.0 & 0.958 & 0.999(0.998,0.999) & 0.778 \\ \text { Hypopnea } & -36.7 \pm 42.7 & 0.915 & 0.914(0.853,0.950) & 0.847 \\ \text { Obstructive Apneas \& Hypopneas } & -40.4 \pm 43.9 & 0.925 & 0.925(0.871,0.957) & 0.847\end{array}$

Mean \pm SD. Kappa statistics comparing montage 3 to montage 1 for sleep apnea severity based on the event index (number of events per hour of sleep) $\geq 5$.

Table 4-Comparison of number of events scored per study in Montage 1 vs. Montage 4

$\begin{array}{lcccc} & \begin{array}{c}\text { Difference (M4 -M1) } \\ (\text { mean } \pm \text { SD) }\end{array} & \begin{array}{c}\text { Spearman } \\ \text { correlation }\end{array} & \begin{array}{c}\text { ICC } \\ (95 \% \text { Cl) }\end{array} & \begin{array}{c}\text { Kappa } \\ \text { statistic }\end{array} \\ \text { Central Apnea } & 0.8 \pm 2.5 & 0.925 & 0.998(0.997,0.999) & 0.693 \\ \text { Hypopnea } & -38.0 \pm 59.6 & 0.863 & 0.841(0.736,0.906) & 0.502 \\ \text { Obstructive Apneas \& Hypopneas } & -55.5 \pm 46.2 & 0.914 & 0.914(0.854,0.950) & 0.563\end{array}$

Mean \pm SD. Kappa statistics comparing montage 4 to montage 1 for sleep apnea severity based on the event index (number of events per hour of sleep) $\geq 5$.

of events scored per study when using montage 2 . The overall levels of agreement for scoring obstructive apneas, central apneas, hypopneas, and total events all exceeded 0.95 ; similarly, Spearman correlation also demonstrated high levels of agreement in different event scoring. Bland-Altman plots did not show a systematic bias in scoring. Bland-Altman plots for montages 1 and 2 (i.e., identifying events using RIP vs. $\mathrm{PVDFb}$ ) are shown in Figures 2A and 2B for obstructive apneas and hypopneas, respectively. Kappa statistics comparing montage 2 to montage 1 for obstructive apnea and total events were slightly lower but still excellent at 0.935 and 0.847 , respectively.

\section{Comparison of Montage 1 and 3: Influence of Using PVDFb without NPT vs. RIP with NPT}

Montage III included only thermistry and PVDFb for breathing assessment. Thus, a comparison of Montages 3 and 1 assesses the comparability of using PVDFb alone for hypopnea detection compared to the reference standard, which includes both RIP and NPT. As shown in Table 3, the overall level of agreement for scoring each event subtype was high. Specifically, for hypopnea scoring, the ICC was $0.914(0.853$, 0.950 ; IQR). The mean difference in the identification of hypopnea using each method was $36.7 \pm 43$.9. Thus for an average recording of $7.1 \mathrm{~h}$, the elimination of NPT would result in a reduction of 5.6 hypopneas per hour. Using a dichotomous cutoff to identify disease yielded an excellent level of agreement $(\kappa=0.847)$.

\section{Comparison of Montage 1 and 4: Influence of Using PVDFb Alone for Respiratory Scoring}

Montage 4 included PVDFb only and when compared with montage 1 , scoring assesses the ability to detect total disordered breathing events against the reference standard of using NPT, thermistry, and RIP. The average number of events was significantly lower for M4 than M1 (Table 1). For the dichotomous outcome of sleep apnea, there was only a moderate level of agreement $(\kappa=0.563)$ (Table 4). However, a high level of agreement was observed for total number and number of central and hypopnea events when evaluated using the ICC.

\section{Intrascorer Reliability}

Intrascorer reliability was assessed for montages 1 to 4 for 30 randomly chosen studies (Table 5). The ICCs for all events exceeded 0.96 for all montages.

\section{Signal Quality}

Mean signal grade for all sensors was above 4.5, with no signal having more frequent poor grades than others. For example, the mean signal quality grade for PVDFb was $4.8 \pm 0.4$ (range 3 to 5) and for RIP signal grade was $4.8 \pm 0.5$ (range 3 to 5).

\section{Study 2: Comparisons to the PNT Reference Standard Sensor}

Total number of events detected by PNT, RIP, and PVDFb numbered 335, 277, and 272, respectively. Mean number of detected events per subject for PNT, RIP, and PVDFb were 
Figure 2-Bland-Altman plot showing the association between the number of respiratory events identified using montages 1 and 2

A

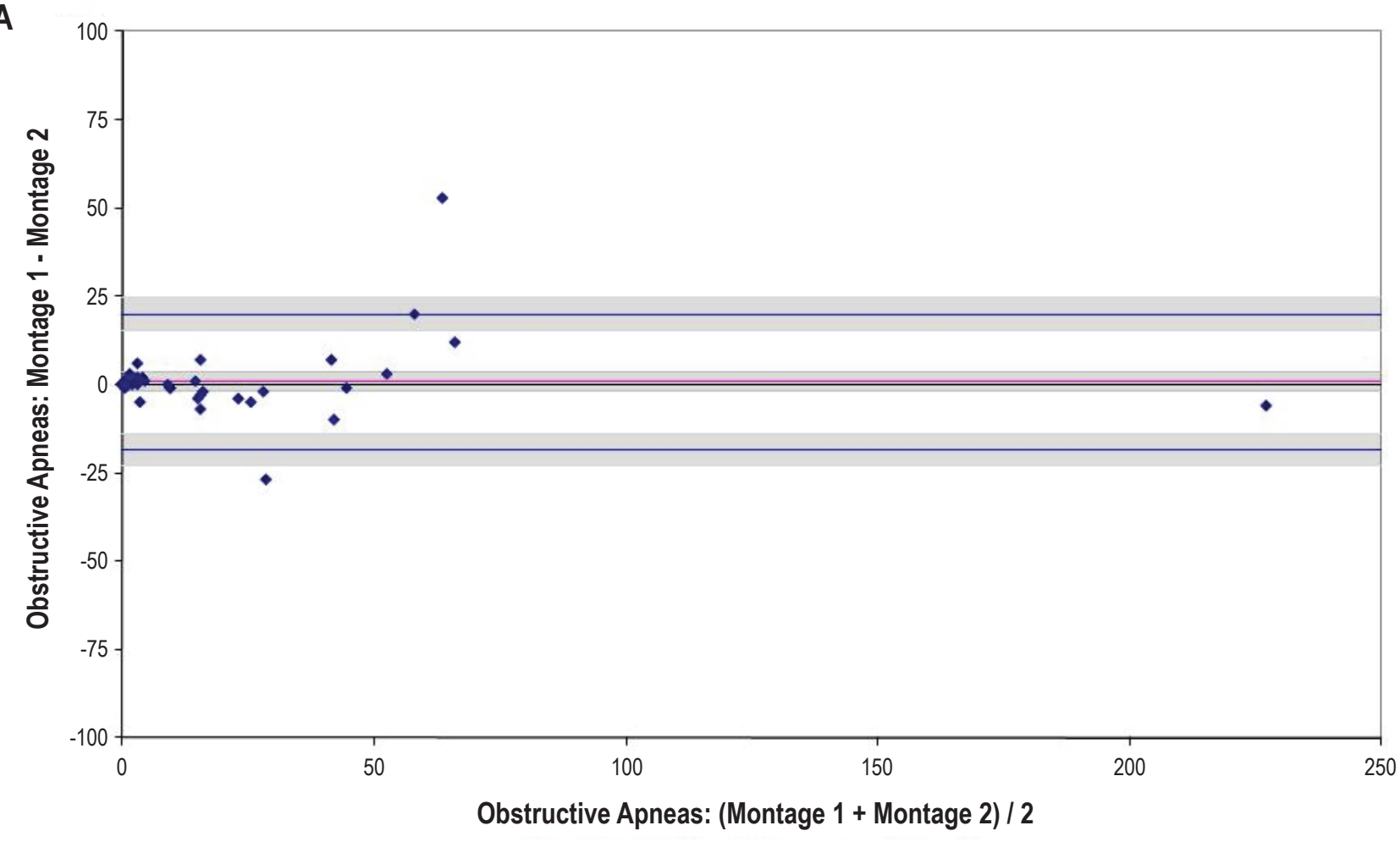

B

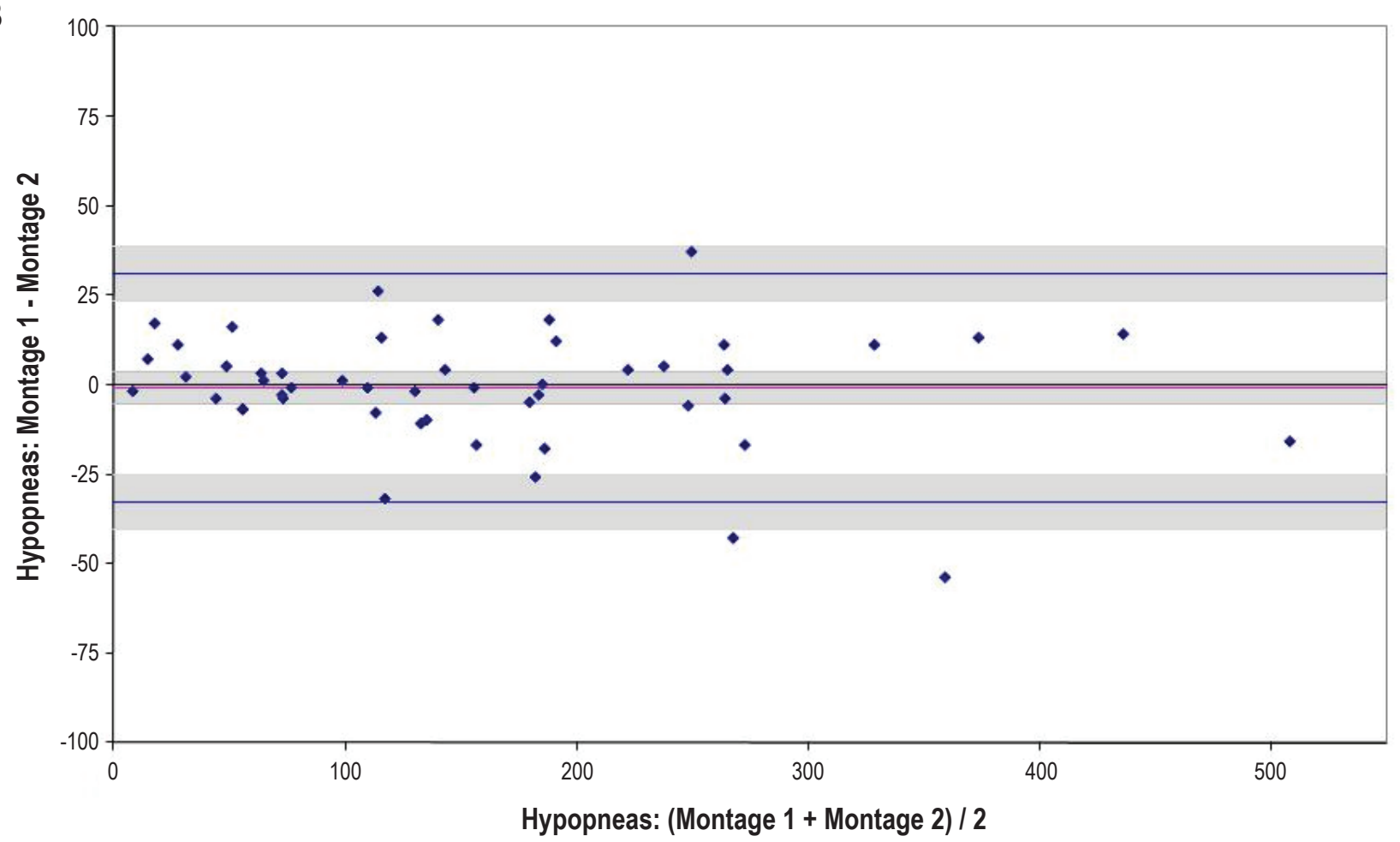

(A) Shows obstructive apneas and (B) shows hypopneas. Y-axis shows the difference in number of events using different montages; the $x$-axis shows the mean number of events identified using the two montages. The horizontal lines reflect the mean bias and the limits of agreement, and shaded bands represent $95 \%$ confidence intervals around each. For obstructive apneas (A), the mean bias (and $95 \% \mathrm{Cl}$ ) was $0.80(-1.86,3.46)$, the lower limit (mean bias -2 standard deviations) was -18.37 (-22.97, -13.77), and the upper limit (mean bias +2 standard deviations) was $19.97(15.37,24.57)$. For hypopneas (B), the mean bias was $-0.92(-5.36,3.52)$, the lower limit (mean bias -2 standard deviations) was $-32.93(-40.61,-25.25)$, and the upper limit (mean bias +2 standard deviations) was $31.09(23.41,38.77)$. 
Table 5-Intrascorer reliability data

\begin{tabular}{|c|c|c|c|c|c|c|}
\hline & \multicolumn{2}{|c|}{ Montage 1} & \multicolumn{2}{|c|}{ Montage 2} & \multicolumn{2}{|c|}{ Montage 3} \\
\hline & $\begin{array}{l}\text { Spearman } \\
\text { Correlation }\end{array}$ & ICC (95\% Cl) & $\begin{array}{l}\text { Spearman } \\
\text { Correlation }\end{array}$ & ICC (95\% CI) & $\begin{array}{l}\text { Spearman } \\
\text { Correlation }\end{array}$ & ICC (95\% Cl) \\
\hline Obstructive apnea & 0.987 & $0.98(0.92,0.99)$ & 1.000 & $0.97(0.90,0.99)$ & 1.000 & $0.99(0.99,1.0)$ \\
\hline Central apnea & 1.000 & $0.99(0.98,0.99)$ & 1.000 & $0.99(0.99,0.99)$ & 0.664 & $0.96(0.85,0.99)$ \\
\hline Hypopnea & 1.000 & $0.99(0.99,1.0)$ & 0.939 & $0.98(0.94,0.99)$ & 0.854 & $0.96(0.84,0.99)$ \\
\hline Total Events & 1.000 & $0.99(0.99,1.0)$ & 0.939 & $0.98(0.95,0.99)$ & 0.951 & $0.97(0.89,0.99)$ \\
\hline
\end{tabular}

Table 6-Number of scored respiratory events by sensor during wake breathing trials

\begin{tabular}{|c|c|c|c|}
\hline & PNT & RIP & PVDFb \\
\hline رen & $33.5 \pm 6.6$ & $27.7 \pm 3.9 \quad P=0.03$ & $27.2 \pm 4.6 \quad P=0.004$ \\
\hline
\end{tabular}

Mean \pm SD. P-value from Wilcoxon test comparing to PNT.

$33.5 \pm 6.6,27.7 \pm 3.9$, and $27.2 \pm 4.6$, respectively (Table 6). Both RIP and PVDFb detected significantly fewer events than did PNT (p-values of 0.03 and 0.004 , respectively). RIP and $\mathrm{PVDFb}$ did not differ in the number of respiratory events detected $(27.7 \pm 3.9$ vs. $27.2 \pm 4.6 ; \mathrm{p}=0.89)$.

\section{DISCUSSION}

In this study, we followed current recommendations in sensor evaluation by systematically comparing a new sensor designed to detect respiratory effort, the PVDFb, to several standard or "reference-standard" comparison groups. During sleep and in a usual clinical laboratory setting, we compared scoring results when the PVDFb was substituted for RIP in a montage which was otherwise consistent with current laboratory sensor use recommendations (Montage 1 vs. 2). We also assessed the comparability of event identification when using a more reduced set of respiratory channels that included PVDFb but did not include other standard sensors, such as NPT and thermistry. Finally, we evaluated the comparability of PVDFb and RIP compared to the reference standard pneumotachography for event identification from data collected during a simulated wake breathing experiment. Our results show that in a typical clinical sleep laboratory setting, PVDF impedance belts are comparable to RIP in identifying effort changes needed to distinguish central from obstructive apneas. Classification of apnea into central and obstructive was almost identical using the two different technologies, with ICCs for the central and obstructive apnea being 0.999 and 0.964 , respectively. Further evidence for the utility of PVDFb sensors in clinical practice are supported by: (1) the comparability of overall event detection for PVDFb and RIP when each were compared to reference standard measurement with pneumotachography; (2) the high quality of signals (little artifact) for PVDFb; and (3) the high intrascorer reliability for event detection using PVDFb within several different scoring montages.

Given the pressures to simplify recording montages, we were also interested in whether reduced montages that included PVDFb but did not include NPT and/or thermistry could be used to accurately score respiratory events. We found a high level of agreement, albeit a modest underestimation for the number of events scored by PVDFb alone (without use of NPT and/or thermistry) compared to scoring using a full montage. Similarly, both PVDFb and RIP modestly underestimated breathing events when compared to the reference standard PNT. These data are consistent with prior research showing the overall greater sensitivity of flow and pressure measurements compared to changes in respiratory excursions in detecting events. Our finding of general comparability of RIP and PNT is similar to conclusions of earlier studies. ${ }^{17-19}$

Polysomnography is the current reference standard for the diagnosis of sleep disordered breathing. Technology that produces signals that accurately reflect respiratory flow and effort while remaining free of artifact is considered fundamental for reliable identification of abnormal respiratory events. Thermal sensors and nasal pressure transducers are the AASM recommended reference standard in the identification of apnea and hypopnea, respectively. Studies have shown that nasal pressure transduction agrees well with reference standard pneumotachography in the identification of apnea and hypopnea. ${ }^{18,19}$ Thermistry has not proven to be a reliable measure of airflow, although, because of the high sensitivity and nonlinear response characteristics of the PNT, thermistry has been endorsed to distinguish obstructive apneas from hypopneas. ${ }^{20,21}$ Currently, the AASM also recommends the use of RIP to determine breathing effort and as an alternative to NPT for hypopnea detection. ${ }^{7}$

Accurate assessment of respiratory effort is needed to correctly classify apnea as obstructive or central. The reference standard in measuring respiratory effort is esophageal manometry which reflects changes in intrathoracic pressure. ${ }^{22}$ However, the technology requires that a catheter be placed into the esophagus through the nose, which can adversely affect sleep state. ${ }^{23}$ Electromyography of intercostal muscles may also be used to determine breathing effort; however, there is some concern for nonlinearity of signal with mouth pressure. ${ }^{24}$ Most commonly in sleep laboratories, respiratory inductance plethysmography (RIP) is employed to estimate the effort of breathing.

The current study demonstrates that both technologies, RIP and PVDF, when incorporated into a belt reflect respiratory effort to a comparable degree. The study strengths include the use of clearly defined approaches for comparing the new sensor to 
the reference standard, both in a typical laboratory setting and in an experimental setting that allowed measurement against a quantitative reference standard assessment of flow and pressure. In addition to agreement, we assessed signal quality and scorer reliability, issues relevant to the successful use of technology in clinical settings. In interpreting the findings, it is important to note that scoring was performed by a highly trained research polysomnologists, and the high scoring reliability we found may be higher than in the usual clinical setting. A study limitation was that assessment of comparability to pneumotachography was performed during simulated wake testing periods due to the problem of having subjects sleep while using multiple sensors that included a face mask. Additionally, the evaluation of the comparability of sensors in the PNT study was limited to event identification rather than a quantitative assessment of volume changes, as volume calibration for both PVDFb and RIP is impractical. The latter was due to challenges in precisely aligning the output of the RIP, PVDFb, and PNT due to differing time constants for each of these sensors. Further, the frequency and phase shift differences between an inductance-based sensor (RIP) and an impedance-based (PVDF) sensor made it difficult to normalize sensor output in a way that permitted comparison of absolute signal values. The study population had an AHI distribution reflective of many laboratories (only $25 \%$ with an AHI $<15$ ), thus limiting the ability to generalize to studies on mildly affected individuals.

\section{CONCLUSIONS}

PVDFb was comparable to standard RIP in determining respiratory effort during polysomnography. When incorporated into otherwise standardized montages that include NPT and thermistry, nearly identical numbers of each respiratory subtype were identified, suggesting it can be used as an alternative to RIP for evaluation of apneas and hypopneas. Further, the comparability of PVDFb to RIP suggests that PVDFb may be used just as RIP often is, as a "back-up" signal for detecting respiratory events when NPT signals become artifactual or are lost. With the ongoing evolution of technology, there needs to be ongoing consideration of what critical sensors should be minimally used to adequately evaluate sleep disordered breathing, as economic and other practical concerns require the adoption of reliable, cost-effective diagnostic strategies for the large pool of patients with undiagnosed sleep apnea.

\section{REFERENCES}

1. Young T, Palta M, Dempsey J, Skatrud J, Weber S, Badr S. The occurrence of sleep-disordered breathing among middle-aged adults. $N$ Engl $\mathrm{J}$ Med 1993;328:1230-5.

2. Goncalves MA, Paiva T, Ramos E, Guilleminault C. Obstructive sleep apnea syndrome, sleepiness, and quality of life. Chest 2004;125:2091-6.

3. Young T, Finn L, Peppard PE, et al. Sleep disordered breathing and mortality: eighteen-year follow-up of the Wisconsin sleep cohort. Sleep 2008;31:1071-8.

4. Shahar E, Whitney CW, Redline S. Sleep-disordered breathing and cardiovascular disease: cross sectional results of the Sleep Heart Health Study. Am J Respir Crit Care 2001;163:19-25.

5. Punjabi NM, Caffo BS, Goodwin JL, et al. Sleep-disordered breathing and mortality: a prospective cohort study. PLoS Med 2009;6:e1000132.

6. Redline S, Budhiraja R, Kapur V, et al. The scoring of respiratory events in sleep: reliability and validity. J Clin Sleep Med 2007;3:169-200.
7. Iber C, Ancoli-Israel, Sonia, Chesson A, Quan SF. AASM manual for the scoring of sleep and associated events: rules, terminology and technical specifications, $1^{\text {st }} \mathrm{ed}$.: Westchester, IL: American Academy of Sleep Medicine, 2007.

8. Masa JF, Corral J, Martín MJ, et al. Assessment of thoracoabdominal bands to detect respiratory effort-related arousal. Eur Resp J 2003;22:661-667.

9. Tasaki I, Iwasa K. Temperature changes associated with nerve excitation: detection by using polyvinylidene fluoride film. Biochem Biophys Res Commun 1981;101:172-6.

10. Fukada E. History and recent progress in piezoelectric polymers. IEEE Trans Ultrason Ferroelectr Freq Control 2000;47:1277-90.

11. Karr SG, Karwoski T, Jacobs JE, Mockros LF. Transducer system for the noninvasive recording of arterial pressure contours. Ann Biomed Eng 1985;13:425-42.

12. Kennelly AE. Impedance. IEEE 1893.

13. Lorenz L. Über die Fortpflanzung der Elektrizität. Annalen der Physik 1879;7:161-93

14. Cohen KP, Ladd WM, Beams DM, Sheers WS, Radwin RG, Tompkins WJ, Webster JG. Comparison of impedance and inductance ventilation sensors on adults during breathing, motion, and simulated airway obstruction. IEEE Trans Biomed Eng 1997;44:555-66.

15. American Academy of Sleep Medicine. EEG arousals: scoring rules and examples; a preliminary report from the Sleep Disorders Atlas Task Force of the American Sleep Disorders Association. Sleep 1992;1:51-7.

16. Redline S, Sanders MH, Lind BK et al. Methods for obtaining and analyzing unattended polysomnography data for a multicenter study. Sleep Heart Health Research Group. Sleep 1998;21:759-67.

17. Cantineau JP, Escourrou P, Sartene R, Gaultier C, Goldman M. Accuracy of respiratory inductive plethysmography during wakefulness and sleep in patients with obstructive sleep apnea. Chest 1992;102:1145-51.

18. Heitman SJ, Atkar RS, Hajduk EA, Wanner RA, Flemons WW. Validation of nasal pressure for the identification of apneas/hypopneas during sleep. Am J Respir Crit Care Med 2002:166:386-91.

19. Thurnheer R, Xie X, Bloch KE. Accuracy of nasal cannula pressure recordings for assessment of ventilation during sleep. Am J Respir Crit Care Med 2001;164:1914-9

20. Berg S, Haight JS, Yap V, Hoffstein V, Cole P. Comparison of direct and indirect measurements of respiratory airflow: implications for hypopneas. Sleep 1997;20:60-64

21. Farre R, Montserrat JM, Rotger M, Ballester E, Navajas D. Accuracy of thermistors and thermocouples as flow-measuring devices for detecting hypopnoeas. Eur Respir J 1998;11:179-82.

22. Milic-Emili J, Mead J, Turner JM, Glauser EM. Improved technique for estimating pleural pressure from esophageal balloons. J Appl Physiol 1964; 19:207-11.

23. Chervin RD, Aldrich $\mathrm{S}$. Effects of esophageal pressure monitoring on sleep architecture. Am J Respir Crit Care Med 1997;156:881-5.

24. Ng GY, Stokes MJ. Relationship between inspiratory mouth pressure and respiratory muscle activity in normal subjects. Respir Med 1992;86:305-9.

\section{ACKNOWLEDGMENTS}

The authors thank Belinda Gray for her help in identifying subjects and Daniel Laskowski for his help with the pneumotachographic technology.

\section{SUBMISSION \& CORRESPONDENCE INFORMATION}

Submitted for publication January, 2011

Submitted in final revised form June, 2011

Accepted for publication June, 2011

Address correspondence to: Brian Koo, Department of Neurology, University Hospitals Case Medical Center, 11100 Euclid Avenue, Cleveland, OH 44120; Tel: (216) 844-3422; Fax: (216) 983-0792; E-mail: koobri@gmail.com

\section{DISCLOSURE STATEMENT}

This study was funded by Dymedix Corporation. Dr. Redline has received equipment from Philips Respironics for research studies. She is the first incumbent of an endowed professorship donated to the Harvard Medical School by Dr. P. Farrell, the founder and Board Chairman of ResMed, through a charitable trust instrument, with equal support equivalent to the endowment payout provided to Harvard Medical School during Dr. Farrell's lifetime by the ResMed Company through an irrevocable gift agreement. The other authors have indicated no other financial conflicts of interest. 